\title{
Constraints on Dbar uplifts
}

\section{S.P. deAlwis}

Physics Department, University of Colorado, Boulder, CO 80309 U.S.A.

E-mail: dealwiss@colorado.edu

ABSTRACT: We discuss constraints on KKLT/KKLMMT and LVS scenarios that use antibranes to get an uplift to a deSitter vacuum, coming from requiring the validity of an effective field theory description of the physics. We find these are not always satisfied or are hard to satisfy.

KeYWords: Strings and branes phenomenology, Supersymmetry Phenomenology

ARXIV EPRINT: 1605.06456 
Flux compactifications of type IIB string theory (for reviews see [1, 2]), have yielded a concrete framework within which to discuss beyond the standard model phenomenology and cosmology. The most popular scenarios involve a first stage with an Anti-deSitter minimum for the moduli potential [3, 4] which is then commonly raised to a deSitter minimum by introducing one or more anti-D3 branes at the bottom of a warped throat in the internal space geometry as in [3]. ${ }^{1}$ In this note we discuss the validity of certain cosmological scenarios within this framework and estimate the one-loop quantum corrections to soft masses, which in principle could be large due to the fact that the SUSY breaking scale is effectively above the ultra-violet (UV) cutoff.

The SUGRA potential in the presence of a D-bar brane (down a warped throat) is of the form

$$
\begin{aligned}
V(\tau, \Phi) & =|F(\tau, \Phi)|^{2}-3 m_{3 / 2}^{2}(\tau, \Phi) M_{P}^{2}+n V_{\bar{D} 3}^{(w)}(\tau, \Phi) \\
& =\mid F(\tau, \Phi))\left.\right|^{2}-3 m_{3 / 2}^{2}(\tau, \Phi) M_{P}^{2}+\left(M_{s}^{(w)}(\tau, \Phi)\right)^{4}+\ldots
\end{aligned}
$$

Here $F$ stands for the F-terms of the moduli and $m_{3 / 2}(\tau, \Phi)$ is the moduli dependent gravitino mass and $\tau$ volume modulus. Also $n$ is the number of D3-bar branes each with potential $V_{\bar{D} 3}^{(w)}$, the superscript $w$ indicating the warping. In the ground state of the Dbar brane this potential is just the fourth power of $M_{s}^{(w)}$ the warped string scale. ${ }^{2}$ Note that such a term is absent if only D branes (and orientifold planes) are present.

This potential makes sense only below the lightest Kaluza-Klein (KK) scale in the problem which is the scale set by KK modes localized at the end of the warped throat. This is necessarily parametrically smaller than the warped string scale, since all effective field theory (EFT) discussions in string theory, depend on proceeding via 10D SUGRA (after freezing string modes). i.e. we need to ensure,

$$
V(\tau, \Phi) \ll\left(M_{\mathrm{KK}}^{(w)}(\tau)\right)^{4} \ll\left(M_{s}^{(w)}(\tau)^{4} .\right.
$$

In the literature on string inflation the only necessary criterion that is usually imposed is that the Hubble scale is well below the (warped) KK scale i.e. $H \ll M_{\mathrm{KK}}^{(w)}$. However the EFT must describe not only the inflationary regime but also the dynamics at the end of inflation - namely the reheating phase. It is easy to see though that if the scale of the inflationary potential is higher than the (warped) KK scale then this will lead to kinetic energies of the inflaton(s) that is higher than the (warped) KK scale, thus violating the criterion under which the derivative expansion is valid. ${ }^{3}$

One can certainly ensure this at the minimum of the potential - indeed that is the role of the last (uplift) term (which is greater than the warped KK scale)

$$
\left.V_{\min }=V\left(\tau_{0}, \Phi_{0}\right)=\mid F\left(\tau_{0}, \Phi_{0}\right)\right)\left.\right|^{2}-3 m_{3 / 2}^{2}\left(\tau_{0}, \Phi_{0}\right) M_{P}^{2}+\left(M_{s}^{(w)}\left(\tau_{0}, \Phi_{0}\right)\right)^{4} \gtrsim 0 .
$$

However it is not possible to ensure this away from the minimum without functional finetuning. As one moves away from the minimum it becomes harder and harder to satisfy the

\footnotetext{
${ }^{1}$ There are of course exceptions - for a recent comprehensive review of string cosmology see [5].

${ }^{2}$ Note that the 10D equations determine the warp factor at the end of the throat in terms of the complex structure moduli.

${ }^{3}$ See for example section 2.1 and footnote 6 of [6].
} 
bound (2) since the scale of the potential is set by the warped string scale which should be much larger than the warped KK scale (see below for estimates of these).

From the above it appears that there is no consistent EFT picture of inflation in the presence of D-bar uplifts, when the inflaton is one of the fields in the EFT. The LVS case with so-called "Fibre inflation" is however an exception and will be discussed later.

On the other hand inflationary dynamics in constructions (such as KKLT, KKLMMT ${ }^{4}$ ) typically involve the motion of branes moving down the warped throat with inflation ending and a reheating phase taking over once the brane and anti-brane annihilate. Such a process will end with the creation of (light warped) string states and KK states with masses respectively $M_{s}^{(w)}$ and $M_{\mathrm{KK}}^{(w)}$. But this is clearly a stringy process taking place at energy scales $E \sim M_{s}^{(w)} \gg M_{\mathrm{KK}}^{(w)}$. Clearly one cannot discuss this within a $4 \mathrm{D}$ low energy effective field theory. In this case the above potential is just used to establish the background field configuration in which this intrinsically stringy process takes place.

To incorporate warping we adopt the following parametrization of the $10 \mathrm{D}$ string metric. This is related to the $4 \mathrm{D}$ Einstein metric $g_{\mu \nu}$ by $^{5}$

$$
\begin{aligned}
d s^{2}= & G_{M N} d X^{M} d X^{N} \\
= & e^{\phi / 2}\left[e^{-6 u(x)}\left(1+\frac{e^{-4 A(y)}}{e^{4 u(x)}}\right)^{-1 / 2} g_{\mu \nu}(x) d x^{\mu} d x^{\nu}+\right. \\
& \left.e^{2 u(x)}\left(1+\frac{e^{-4 A(y)}}{e^{4 u(x)}}\right)^{1 / 6} \hat{g}_{m n}(y) d y^{m} d y^{n}\right]
\end{aligned}
$$

Here $\phi$ is the dilaton, $g_{m n}$ is a fiducial metric on the internal manifold $X$ which is conveniently normalized such that $\int_{X} \sqrt{\hat{g}} d^{6} y=(2 \pi)^{6} \alpha^{\prime 3}$. So we have (using the relation $2 \kappa_{10}^{2}=(2 \pi)^{7} \alpha^{\prime 4}$ between the $10 \mathrm{D}$ gravitational constant and the string scale in the string metric [10])

$$
\frac{1}{(2 \pi)^{7} \alpha^{4}} \int d^{10} X \sqrt{|G|} e^{-2 \phi} G^{\mu \nu} R_{\mu \nu}(G)+\ldots=\frac{1}{2 \pi \alpha^{\prime}} \int d^{4} x \sqrt{|g(x)|} g^{\mu \nu} R_{\mu \nu}(g)+\ldots
$$

The Planck length $l_{P}$ measured in the Einstein frame is given by $l_{P}^{2}=M_{P}^{-2}=2 \pi \alpha^{\prime} / 2$. Outside of throat regions the warping can be ignored so that $e^{4 u} \gg e^{-4 A}$ and the metric becomes the standard $4 \mathrm{D}$ Einstein metric as given for example in GKP [11]. The volume modulus is then $\tau=e^{4 u(x)}$ so that the dimensionless volume of $X$ is given by $\mathcal{V}=e^{6 u}$. Even in the case where there is one (or more) warped throat, as long as the volume of warped regions is negligible compared to the overall volume, we may still take $\mathcal{V}=e^{6 u(x)} \int d^{6} y\left(1+\frac{e^{-4 A(y)}}{e^{4 y(x)}}\right)^{1 / 6} /(2 \pi)^{6} \alpha^{\prime 3} \simeq \tau^{3 / 2}=e^{6 u}$. However at the bottom of a long warped throat $\left.y \rightarrow y_{0}\right)$ where $e^{-4 A\left(y_{0}\right)} \gg e^{4 u}$ the metric (3) becomes

\footnotetext{
${ }^{4}$ For a recent review with a comprehensive discussion of all the issues involving this type of scenario see Baumann and McAllister [5].

${ }^{5}$ Note that the standard factorized metric used in GKP cannot be used everywhere on the ten dimensional space. In addition in the presence of warping one needs to keep non diagonal terms $G_{\mu n}$ in the metric ansatz - since otherwise the Einstein equation $R_{\mu n}=0$ leads to an overconstrained system as first observed in [7] - see also $[8,9]$.
} 
$d s^{2}=e^{\phi / 2}\left[e^{-4 u} e^{2 A} g_{\mu \nu} d x^{\mu} d x^{\nu}+e^{4 u / 3} e^{-2 A(y) / 3} \hat{g}_{m n}(y) d y^{m} d y^{n}\right]$. The effective KK radius depends on the location in the internal space. Thus this parametrization provides an interpolation between the unwarped bulk region and the warped throat region as discussed in [12].

The string scale for strings located at a fixed value of the internal coordinate $y$ can be read off from the string action which reads (using (3)),

$$
\begin{aligned}
\frac{1}{2} \frac{1}{2 \pi \alpha^{\prime}} \int_{\mathrm{ws}} & d^{2} \sigma \sqrt{\operatorname{det}\left(G_{M N} \partial_{\alpha} X^{M} \partial_{\beta} X^{N}\right)} \\
= & \frac{1}{2} \frac{1}{2 \pi \alpha^{\prime}} \int_{\mathrm{ws}} d^{2} \sigma e^{\phi(x(\sigma), y) / 2} e^{-6 u(x(\sigma)}\left(1+\frac{e^{-4 A(y)}}{e^{4 u(x(\sigma))}}\right)^{-1 / 2} \\
& \times \sqrt{\operatorname{det}\left(g_{\mu \nu} \partial_{\alpha} x^{\mu}(\sigma) \partial_{\beta} x^{\nu}(\sigma)\right)}
\end{aligned}
$$

so that relative to the $4 \mathrm{D}$ Einstein metric the string scale is (with \langle\rangle$_{\mathrm{ws}}$ denoting average over the world sheet),

$$
M_{s}^{2}=\frac{1}{2 \pi \alpha^{\prime}}<e^{\phi / 2} e^{-6 u(x)}\left(1+\frac{e^{-4 A(y)}}{e^{4 u(x(\sigma))}}\right)^{-1 / 2}>_{\mathrm{ws}} .
$$

Thus we have in the different regions:

$$
\begin{aligned}
M_{s}^{2} & =\frac{1}{2 \pi \alpha^{\prime}} e^{\phi / 2} e^{-6 u(x)}=M_{P}^{2} \frac{e^{\phi / 2}}{2 \mathcal{V}}, & & \text { unwarped } \\
M_{s}^{(\mathrm{W}) 2} & =\frac{e^{\phi / 2}}{2 \pi \alpha^{\prime}} \mathrm{e}^{-4 \mathrm{u}(\mathrm{x})} \mathrm{e}^{2 \mathrm{~A}(\mathrm{y})}=\mathrm{M}_{\mathrm{P}}^{2} \frac{\mathrm{e}^{\phi / 2}}{2 \mathcal{V}^{2 / 3}} \mathrm{e}^{2 \mathrm{~A}(\mathrm{y})} . & & \text { warped KKLMMT }
\end{aligned}
$$

The KK mass matrix turns out to be, after expanding $\Phi(x, y)=\sum \phi_{r}(x) \omega_{s}(y)$ where $\omega_{r}(y)$ is an orthonormal (in the metric $\hat{g}$ ) basis of harmonics on $X$,

$$
M_{\mathrm{KKrs}}^{2}=\frac{1}{(2 \pi)^{7} \alpha^{4}} \int_{X} d^{6} y \sqrt{\hat{g}} e^{-8 u(x)}\left(1+\frac{e^{-4 A(y)}}{e^{4 u(x)}}\right)^{-2 / 3} \hat{g}^{m n}(y) \partial_{m} \omega_{r}(y) \partial_{n} \omega_{s}(y) .
$$

Thus we have in the different regions, ignoring an $O(1)$ matrix;

$$
\begin{aligned}
M_{\mathrm{KK}}^{2} & =\frac{1}{2 \pi \alpha^{\prime}} e^{-8 u(x)}=\frac{M_{P}^{2}}{2 \mathcal{V}^{4 / 3}}, & & \text { unwarped } \\
M_{\mathrm{KK}}^{(\mathrm{W}) 2} & =\frac{1}{2 \pi \alpha^{\prime}} e^{-16 u(x) / 3} e^{8 A\left(y_{0}\right) / 3}=\frac{M_{P}^{2} e^{8 A\left(y_{0}\right) / 3}}{2 \mathcal{V}^{8 / 9}}, & & \text { warped KKLMMT. }
\end{aligned}
$$

Note that the last two relations applies to modes $\omega_{r}(y)$ that have support in a strongly warped throat $y \sim y_{0}$. We observe also that

$$
\frac{M_{\mathrm{KK}}^{2}}{M_{s}^{2}}=\frac{e^{-\phi / 2}}{\mathcal{V}^{1 / 3}} ; \frac{M_{\mathrm{KK}}^{(\mathrm{W}) 2}}{M_{s}^{(\mathrm{W}) 2}}=\frac{e^{-\phi / 2} e^{2 A\left(y_{0}\right) / 3}}{\mathcal{V}^{2 / 9}} .
$$

The last two relations apply to the unwarped and warped (KKLMMT) cases respectively and clearly both satisfy the necessary criterion that the KK scale should be well below the 
string scale for large volume and warping. The requirement that the gravitino mass is less than the (warped) KK then gives (using $m_{3 / 2}^{2}=g\left|W_{0}\right|^{2} M_{P}^{2} / \mathcal{V}^{2}$ and eqn (9))

$$
\frac{g^{3 / 2}\left|W_{0}\right|^{3}}{\mathcal{V}^{5 / 3}} \ll e^{4 A\left(y_{0}\right)} \ll \frac{1}{\mathcal{V}^{2 / 3}},
$$

where the second relation is the statement of being in the region of large warping (see discussion below eqn (4).

For the corrected (KKLMMT) KKLT stabilization mechanism where the supersymmetric AdS minimum is uplifted to Minkowski/dS space, the relevant uplift condition gives

$$
3 M_{P}^{4} \frac{e^{\phi}\left|W_{0}\right|^{2}}{\mathcal{V}^{2}} \simeq M_{s}^{(W) 4}=M_{P}^{4} \frac{e^{\phi}}{4 \mathcal{V}^{4 / 3}} e^{4 A\left(y_{0}\right)}
$$

leading to

$$
e^{4 A\left(y_{0}\right)}=12 \frac{W_{0}^{2}}{\mathcal{V}_{0}^{2 / 3}} \ll 1 .
$$

Let us now discuss whether adding an anti-brane contribution to the potential can give us Minkowski (or dS) space when used in conjunction with the spontaneously broken supersymmetric, albeit AdS, equilibrium (LVS) solution of BBCQ [4]. If the scale of the inflationary potential is given by the warped string scale, as would be the case generically for Dbar uplift theories (see the discussion above eqn (2)), then the same conclusion applies and there is no $4 \mathrm{D}$ EFT that covers inflation and reheating. However unlike in the KKLT case in LVS there is another mechanism for inflation - namely the so-called "Fibre Inflation" model. In this case (for details see $[6,13]$ ) one starts with CY manifolds that are (for instance) K3 fibre bundles. Compared to the original BBCQ class of models there is in addition to the volume modulus, (at least) one more large modulus in addition to the small (exceptional divisor) modulus $\tau_{s}$. The canonical example is $\mathcal{V}=a \sqrt{\tau^{1}} \tau^{2}-b \tau_{s}^{3 / 2}$.

In this case there is at least one flat direction that survives the BBCQ (plus uplift to $\mathrm{dS}$ space) stabilization procedure, that can be used to get an inflationary scenario. This is stabilized by adding in string loop and/or $\alpha^{\prime 3}$ terms that result in $F^{4}$ terms. This gives a viable EFT description of inflation satisfying all the observational constraints though predicting very low power in tensor modes. For us the relevant question is whether this mechanism survives the use of anti-branes to accomplish the uplift to dS.

The new element here is that there is a relation in the LVS construction amongst the three terms of (1) coming from the extremization with respect to the small modulus $\tau_{s}$ and the volume $\mathcal{V}$. The inflationary potential then has essentially a fixed value of $\mathcal{V}$ but the important point is that there are cancellations amongst the three terms, so that the scale of inflation is now given by,

$$
V_{\mathrm{inf}} \simeq \frac{g W_{0}^{2} \Phi\left(\ln \mathcal{V} / W_{0}\right)}{\mathcal{V}^{3}} M_{P}^{4} \ll M_{\mathrm{KK}}^{(W) 4}=\frac{e^{16 A\left(y_{0}\right) / 3}}{4 \mathcal{V}^{16 / 9}} M_{P}^{4},
$$

where $\Phi \sim\left(\ln \left(\mathcal{V} / W_{0}\right)\right)^{3 / 2}$ and in the second relation we have again imposed the bound (2). This gives us

$$
e^{4 A_{0}} \gg \frac{\left(g W_{0}^{2} \Phi\right)^{3 / 4}}{\mathcal{V}^{11 / 12}}
$$


On the other hand the LVS (plus Dbar uplift term) stabilization conditions give ${ }^{6}$

$$
\tau_{s}^{3 / 2} \simeq \frac{\xi}{2 \sqrt{g^{3}}}+\frac{16}{27} \frac{e^{4 A_{0}}}{|W|^{2}} \mathcal{V}^{5 / 3} \simeq\left(a^{-1} \ln \frac{\mathcal{V}}{\left|W_{0}\right|}\right)^{3 / 2}
$$

Combining this with the bound (11) we get a bound on the volume,

$$
\left.\mathcal{V} \ll\left(\frac{27}{16}\right)^{4 / 3} \frac{\left|W_{0}\right|^{2 / 3}}{g \Phi\left(\ln \mathcal{V} / W_{0}\right)}\left(a^{-1} \ln \mathcal{V} / W_{0}\right)\right)^{2} \sim \frac{\left|W_{0}\right|^{2 / 3}}{g a^{2}}\left(\ln \frac{\mathcal{V}}{W_{0}}\right)^{1 / 2}
$$

Since typically $W_{0} \lesssim O(1), g \sim 10^{-1}$ in LVS constructions this bound is hard to satisfy for the typically large values $\mathcal{V} \gtrsim 10^{3}$ that are usually required in LVS constructions.

Recently backgrounds involving an anti-brane have been discussed in terms of nonlinearly realized SUSY (see for example [14] and references therein), so that its effect is represented by a Goldstino field. The latter in turn comes from a nilpotent superfield $X$ which satisfies $X^{2}=0$. Adding the contribution of this field to the usual Kaehler and superpotential terms we have ${ }^{7}$ (with $k(U, \bar{U}) \equiv i \int_{X} \Omega \wedge \bar{\Omega}$ ) and $M_{P}=1$ )

$$
\begin{aligned}
& K=-2 \ln \mathcal{V}-\ln (S+\bar{S})-\ln k(U, \bar{U})+\frac{c\left(U, \bar{U}, S_{R}\right)}{\mathcal{V}^{\beta}} X \bar{X}+\ldots, \\
& W=M^{2} X+W_{\text {flux }}+W_{\mathrm{np}}+W_{\mathrm{sm}} .
\end{aligned}
$$

The last term $W_{\mathrm{sm}}$ is the supersymmetric standard model superpotential. The additional term coming from the anti-brane is then, ${ }^{8} M_{P}^{-4}\left|F^{X}\right|^{2}=e^{K} K^{X \bar{X}} M^{4}=\frac{e^{\phi} c^{-1} \mathcal{V}^{\beta}}{2 \mathcal{V}^{2} k(U, \bar{U})} M^{4}$. Comparing $\left|F^{X}\right|^{2}$ with the leading term of the $\bar{D}_{3}$ brane action in the Einstein frame $M_{s}^{w) 4}$ (see (7)) and using the fact that $M$ has to be holomorphic or a constant (since it is a term in the superpotential) we find in the KKLMMT case, $M^{4}=\frac{1}{2 \pi} e^{4 A_{0}}, \beta=2 / 3, c=\frac{e^{\phi}}{k(U, \bar{U})}$. With these identifications the low energy effects of the anti-brane can clearly be represented within the formal context of $\mathcal{N}=1$ SUGRA. ${ }^{9}$

However a formal representation does not imply that the usual properties of linearly realized and spontaneously broken SUSY hold here. Indeed SUSY is broken above the cutoff scale of the EFT. $F_{X} \simeq\left(M_{s}^{(w)}\right)^{2}>\left(M_{\mathrm{KK}}^{(w)}\right)^{2} \equiv \Lambda_{\text {cutoff }}^{2}$. Thus perturbative corrections to masses couplings etc will not have SUSY cancellations in the EFT and will be no different from that in a non-supersymmetric theory cutoff at $M_{\mathrm{KK}}^{(w)}$. In particular one might expect that soft scalar masses (and hence the Higgs mass in particular), will acquire quantum corrections $\Delta m_{0}^{2} \simeq \Lambda_{\text {cutoff }}^{2} / 16 \pi^{2}$ and that the classical calculation has no meaningful phenomenological consequences.

The actual situation is however is somewhat more complicated. It will turn out that although the phenomenology coming from a KKLT type stabilization (with the flux superpotential taking extremely tiny values $\left(W_{0} \sim e^{-\mathcal{V}^{2 / 3}}\right)$ will acquire one-loop corrections

\footnotetext{
${ }^{6} \mathrm{Up}$ to $O(1)$ factors the LVS potential with Dbar uplift term after fixing the complex structure and the dilaton, takes the form, $\frac{4}{3} g(a|A|)^{2} \frac{\sqrt{\tau_{s}} e^{-2 a \tau_{s}}}{\mathcal{V}}-2 g a\left|A W_{0}\right| \frac{\tau_{s} e^{-a \tau_{s}}}{\mathcal{V}^{2}}+\frac{3}{8} \frac{\xi\left|W_{0}\right|^{2}}{g^{1 / 2} \mathcal{V}^{3}}+\frac{g}{2 \mathcal{V}^{4 / 3}} e^{4 A\left(y_{0}\right)}$. The equations (12) come from the extremization conditions $\partial_{\tau^{s}} V=\partial_{\mathcal{\nu}} V=0$.

${ }^{7}$ This is a somewhat modified version of the discussion in [14].

${ }^{8}$ Note that we are taking dimensionless moduli so $K, W, M, X$ are all dimensionless.

${ }^{9}$ For an earlier discussion using spurion instead of nil-potent superfields see [15].
} 
that tend to vitiate the classical calculations, in the LVS case (with $W_{0} \sim O(1)$ ) these corrections are actually suppressed.

From the Coleman-Weinberg formula the largest supersymmetric standard model field dependent contribution to the effective potential is

$$
\Delta V_{1}=\frac{\Lambda^{2}}{16 \pi^{2}} \operatorname{Str} \mathcal{M}^{2}(\Phi, \bar{\Phi})
$$

where $\operatorname{Str} \mathcal{M}^{2}=\sum(-1)^{2 j+1}(2 j+1) \operatorname{tr} m_{j}^{2}(\Phi, \bar{\Phi})$. Here $m_{j}^{2}$ is the mass matrix for states of spin $j$ and $\Phi=\left\{\Phi^{I}\right\}, I=1, \ldots, N_{T o t}$ ) stands for all the fields in the low energy theory below the cutoff $\Lambda$, i.e. in our case all the moduli and the dilaton as well as the matter fields.

Now in a (spontaneously broken) supersymmetric theory (with all states forming complete supermultiplets below the cutoff) we have [16, 17],

$$
\operatorname{Str} \mathcal{M}^{2}=\left(N_{\text {Tot }}-1\right) m_{3 / 2}^{2}(\Phi, \bar{\Phi})-F^{I}\left(\mathcal{R}_{I \bar{J}}+S_{I \bar{J}}\right) F^{\bar{J}},
$$

where $\mathcal{R}_{I \bar{J}}=\partial_{I} \partial_{J} \ln \operatorname{det} K_{M \bar{N}}$ and $S_{I \bar{J}}=-\partial_{I} \partial_{\bar{J}} \ln \operatorname{det} \Re f_{a b}$ with $f_{a b}$ being the gauge coupling superfield. The field dependent gravitino mass is given by $m_{3 / 2}^{2}(\Phi, \bar{\Phi})=e^{K}|W(\Phi)|^{2}$, where $K$ and $W$ are given by (14)(15). For future reference we also write the matter superpotential as

$$
W_{\mathrm{sm}}=\mu_{i j}(U) C^{i} C^{j}+\lambda_{i j k}(U) C^{i} C^{j} C^{k}+O\left(C^{4}\right),
$$

where $C^{i}$ represent the Higgs, lepton and quark superfields.

In this case the correction to the matter field mass matrix is (see for example [18])

$$
\Delta m_{i \bar{j}}^{2}=-\frac{\Lambda^{2}}{16 \pi^{2}}\left(e^{K} D_{i} D_{M} W \mathcal{R}^{M \bar{L}} D_{\bar{j}} D_{\bar{L}} \bar{W}+O\left(\left(m_{3 / 2}^{2}, F\right)\right) .\right.
$$

The only contribution in the above expression that is not proportional to supersymmetry breaking parameters or to the matter fields $C^{i}$ (which will have vanishingly small expectation values) is the $\mu$-term contribution from (17). In other words we have (restoring $M_{P}$ and the dimensionality of $\mu$ ),

$$
\Delta m_{i \bar{j}}^{2}=-\frac{\Lambda^{2}}{16 \pi^{2} M_{P}^{2}}\left(e^{K} \mu_{i k} \bar{\mu}_{\bar{j} l} \mathcal{R}^{k \bar{l}}+O\left(C, m_{3 / 2}^{2}, F\right)\right) .
$$

Since the $\mu$-term needs to be tuned in any case to be of the same order as the soft SUSY breaking masses, the above quantum correction to the squared soft mss is negligible for cut-offs $\Lambda$ which are well below the Planck scale - as is of course the case here.

On the other hand when as here, the supersymmetry breaking is above the cutoff, one might on general grounds expect a much larger quantum (additive) correction to the squared soft mass. The point is that in this case we do not have a complete supermultiplet for the goldstino field $X$. The scalar superpartner is absent. It corresponds to the (would be) modulus field corresponding to the position of the $\bar{D} 3$ brane at the bottom of the throat and which has been removed by the orientifold projection. Thus the supertrace 
Coleman-Weinberg formula (16) will contain an unpartnered contribution $\operatorname{tr} m_{X \bar{X}}^{2}$ giving us an additional contribution to $(16)^{10}$

$$
\Delta_{X} V_{1}=\frac{\Lambda^{2}}{16 \pi^{2}} \operatorname{tr} m_{X \bar{X}}^{2}=\frac{\Lambda^{2}}{16 \pi^{2}} e^{K} D_{X} D_{I} W D_{\bar{X}} D_{\bar{J}} \bar{W} K^{I \bar{J}} K^{X \bar{X}} .
$$

For simplicity we will specialize to the case of one Kaehler modulus $T$ so that $\mathcal{V} \sim \tau^{3 / 2}, \tau=$ $\Re T$, and also ignore the indices on the matter fields $C^{i}$. Thus we rewrite the Kaehler potential (14) as

$$
K=-3 \ln (T+\bar{T})-\ln (S+\bar{S})-\ln k(U, \bar{U})+\frac{a C \bar{C}}{T+\bar{T}}+\frac{c X \bar{X}}{(T+\bar{T})}+\frac{b C \bar{C} X \bar{X}}{(T+\bar{T})^{2}}+\ldots
$$

Here $a, b, c$ are functions of $U, \bar{U}$, and $S$. After some straightforward calculation we find the leading contribution proportional to $C \bar{C}$ to be ${ }^{11}$

$$
\operatorname{tr} m_{X \bar{X}}^{2} \sim \frac{1}{\mathcal{V}^{2}}\left|\frac{\partial \mu^{2}}{\partial U}\right|^{2} K^{U \bar{U}} K^{X \bar{X}} \sim \frac{1}{\mathcal{V}^{2}}\left|\frac{\partial \mu^{2}}{\partial U}\right|^{2} K^{U \bar{U}} \frac{b}{c^{2}} C \bar{C} .
$$

Hence the largest one-loop contribution to the squared soft mass is

$$
\Delta m_{C \bar{C}}^{2} \sim \frac{\Lambda^{2}}{32 \pi^{2}} \frac{1}{\mathcal{V}^{2}}\left|\frac{\partial \mu^{2}}{\partial U}\right|^{2} K^{U \bar{U}} \frac{b}{c^{2}} .
$$

The $\mu$-term at the minimum of the potential (i.e. with $U=U_{0}$ ) needs to be tuned to be of the order of the weak mass scale (or at most $O\left(m_{3 / 2}\right)$ ) so that standard model particles are at the right scale, but in general away from the minimum it is $O(1)$ on the Planck scale. Thus identifying the cutoff with the KK-scale (given in (9)) we have

$$
\Delta m_{C \bar{C}}^{2} \sim \frac{M_{P}^{2}}{32 \pi^{2}} \frac{e^{8 A / 3}}{\mathcal{V}^{8 / 9}} \frac{O(1)}{\mathcal{V}^{2}} \sim \frac{O(1)}{32 \pi^{2}} \frac{m_{3 / 2}^{2}}{W^{2 / 3}} \frac{1}{\mathcal{V}^{4 / 3}}
$$

In the last relation we have used the KKLT uplift condition (10) to estimate the warp factor. The classical squared soft mass in this class of models is $m_{C \bar{C}}^{2} \sim m_{3 / 2}^{2} /\left|\ln m_{3 / 2}\right|$ and requiring that the quantum contribution (20) does not dominate this gives us the condition

$$
\left|\ln m_{3 / 2}\right|<32 \pi^{2} W_{0}^{2 / 3} \mathcal{V}^{4 / 3}
$$

The Dbar brane contribution uplifts the first stage SUSY AdS minimum of KKLT to Minkowski/dS space. This procedure however works only with highly suppressed values of the flux superpotential - indeed the first stage results in $D_{T} W=0$ which in turn gives (writing $W_{\mathrm{np}}=D e^{-a T}$ )

$$
-a D e^{-a T}=3 \frac{W_{0}}{T+\bar{T}}
$$

\footnotetext{
${ }^{10}$ Note that this is a field dependent contribution to the potential which needs to be differentiated with respect to $C, \bar{C}$ to get the corresponding correction to soft terms. Of course on-shell (i.e. at the minimum of the potential) this will vanish as it is the sqared Goldstino mass.

${ }^{11}$ At the minimum of the potential where we expect $C=0$ the contribution to $\operatorname{tr} m_{X \bar{X}}^{2}$ below will of course vanish!
} 
Thus we get $\left|\ln m_{3 / 2}\right| \sim\left|\ln \frac{W_{0}}{\mathcal{V}}\right| \sim a \tau$. Using this and (22) in the bound (21) we have $e^{2 a \tau / 3}<32 \pi^{2} \tau^{5 / 3}$. This restricts the value of the modulus to the range $1 \ll a \tau \sim \tau \lesssim 15$.

In the LVS case however there is no such restriction since the uplift condition is different. Before the uplift the potential $\sim-e^{\phi}\left|W_{0}\right|^{2} / \mathcal{V}^{3}$. Requiring that this is of the same order as the warped string scale raised to the fourth power we have instead of (10) the estimate $e^{4 A_{0}}=\frac{\left|W_{0}\right|^{2}}{\mathcal{V}^{5 / 3}}$. Using this in the first relation of (20) we get $\Delta m_{C \bar{C}}^{2}=\frac{O(1)}{32 \pi^{2}} \frac{m_{3 / 2}^{2}}{W_{0}^{2 / 3}} \frac{1}{\mathcal{V}^{2}}$.

In the unsequestered LVS case [19], one gets soft masses of the same order as in the KKLT case. Requiring again that the quantum correction does not dominate the classical one now gives,

$$
\left|\ln m_{3 / 2}\right| \sim\left|\ln \frac{W_{0}}{\mathcal{V}}\right|<32 \pi^{2}\left|W_{0}\right|^{2 / 3} \mathcal{V}^{2},
$$

which is easily satisfied since unlike in the KKLT case $W_{0}$ is not required to be exponentially suppressed in LVS. In the sequestered scenario [20-22] the classical squared soft mass is suppressed by an extra factor of $\mathcal{V}$ so that the above relation is replaced by

$$
\left|\ln m_{3 / 2}\right| \sim\left|\ln \frac{W_{0}}{\mathcal{V}}\right|<32 \pi^{2}\left|W_{0}\right|^{2 / 3} \mathcal{V}
$$

This is also easily satisfied.

In conclusion we find that with Dbar uplifts the constraints coming from the validity of an EFT description depend on whether one is in the KKLT case or the LVS case. In the first (KKLT) case they indicate there is no EFT which describes inflationary cosmology without functional fine-tuning. It is necessarily a stringy process coming from brane antibrane annihilation with the EFT just determining the background. Also in order for the classical phenomenology to survive one-loop quantum effects, the Kaehler modulus needs to be less than or equal to a number around 15. In the second (LVS) case the constraints are somewhat different. On the one hand it appears that it is difficult to get a viable EFT description of inflation (such as fibre inflation) with Dbar uplifts because it is hard to satisfy (13). However the classical evaluation of soft masses will not be vitiated by large one-loop corrections even for very large values of the Kaehler moduli.

\section{Acknowledgments}

I wish to thank Joe Polchinski and Fernando Quevedo for discussions and an anonymous referee for several useful comments. I also wish to thank the Abdus Salam ICTP for hospitality during the initial stages of this project.

Open Access. This article is distributed under the terms of the Creative Commons Attribution License (CC-BY 4.0), which permits any use, distribution and reproduction in any medium, provided the original author(s) and source are credited.

\section{References}

[1] M. Graña, Flux compactifications in string theory: A Comprehensive review, Phys. Rept. 423 (2006) 91 [hep-th/0509003] [INSPIRE]. 
[2] M.R. Douglas and S. Kachru, Flux compactification, Rev. Mod. Phys. 79 (2007) 733 [hep-th/0610102] [INSPIRE].

[3] S. Kachru, R. Kallosh, A.D. Linde and S.P. Trivedi, De Sitter vacua in string theory, Phys. Rev. D 68 (2003) 046005 [hep-th/0301240] [INSPIRE].

[4] V. Balasubramanian, P. Berglund, J.P. Conlon and F. Quevedo, Systematics of moduli stabilisation in Calabi-Yau flux compactifications, JHEP 03 (2005) 007 [hep-th/0502058] [INSPIRE].

[5] D. Baumann and L. McAllister, Inflation and string theory, Cambridge University Press, Cambridge U.K. (2015) [arXiv:1404.2601].

[6] C.P. Burgess, M. Cicoli, S. de Alwis and F. Quevedo, Robust inflation from fibrous strings, JCAP 05 (2016) 032 [arXiv: 1603.06789] [INSPIRE].

[7] S.P. de Alwis, On potentials from fluxes, Phys. Rev. D 68 (2003) 126001 [hep-th/0307084] [INSPIRE].

[8] S.P. de Alwis, Brane worlds in $5 D$ and warped compactifications in IIB, Phys. Lett. B 603 (2004) 230 [hep-th/0407126] [INSPIRE].

[9] S.B. Giddings and A. Maharana, Dynamics of warped compactifications and the shape of the warped landscape, Phys. Rev. D 73 (2006) 126003 [hep-th/0507158] [INSPIRE].

[10] S.P. de Alwis, A note on brane tension and M-theory, Phys. Lett. B 388 (1996) 291 [hep-th/9607011] [INSPIRE].

[11] S.B. Giddings, S. Kachru and J. Polchinski, Hierarchies from fluxes in string compactifications, Phys. Rev. D 66 (2002) 106006 [hep-th/0105097] [INSPIRE].

[12] S. Kachru, R. Kallosh, A.D. Linde, J.M. Maldacena, L.P. McAllister and S.P. Trivedi, Towards inflation in string theory, JCAP 10 (2003) 013 [hep-th/0308055] [INSPIRE].

[13] M. Cicoli, C.P. Burgess and F. Quevedo, Fibre inflation: observable gravity waves from IIB string compactifications, JCAP 03 (2009) 013 [arXiv:0808.0691] [INSPIRE].

[14] R. Kallosh, F. Quevedo and A.M. Uranga, String theory realizations of the nilpotent goldstino, JHEP 12 (2015) 039 [arXiv:1507.07556] [INSPIRE].

[15] K. Choi, A. Falkowski, H.P. Nilles and M. Olechowski, Soft supersymmetry breaking in KKLT flux compactification, Nucl. Phys. B 718 (2005) 113 [hep-th/0503216] [INSPIRE].

[16] J. Wess and J. Bagger, Supersymmetry and supergravity, Princeton University Press, Princeton U.S.A. (1992).

[17] S. Ferrara, C. Kounnas and F. Zwirner, Mass formulae and natural hierarchy in string effective supergravities, Nucl. Phys. B 429 (1994) 589 [Erratum ibid. B 433 (1995) 255] [hep-th/9405188] [INSPIRE].

[18] K. Choi, J.S. Lee and C. Muñoz, Supergravity radiative effects on soft terms and the $\mu$ term, Phys. Rev. Lett. 80 (1998) 3686 [hep-ph/9709250] [INSPIRE].

[19] J.P. Conlon, F. Quevedo and K. Suruliz, Large-volume flux compactifications: moduli spectrum and D3/D7 soft supersymmetry breaking, JHEP 08 (2005) 007 [hep-th/0505076] [INSPIRE].

[20] R. Blumenhagen, J.P. Conlon, S. Krippendorf, S. Moster and F. Quevedo, SUSY breaking in local string/F-theory models, JHEP 09 (2009) 007 [arXiv:0906.3297] [INSPIRE].

[21] S.P. de Alwis, Classical and quantum SUSY breaking effects in IIB local models, JHEP 03 (2010) 078 [arXiv: 0912. 2950] [inSPIRE].

[22] L. Aparicio, M. Cicoli, S. Krippendorf, A. Maharana, F. Muia and F. Quevedo, Sequestered de Sitter string scenarios: soft-terms, JHEP 11 (2014) 071 [arXiv:1409.1931] [INSPIRE]. 